\title{
Structure and regulation of the Asr gene family in banana
}

\author{
Isabelle M. Henry • Sebastien C. Carpentier • \\ Suzana Pampurova • Anais Van Hoylandt • \\ Bart Panis · Rony Swennen · Serge Remy
}

Received: 23 December 2010/Accepted: 17 April 2011/Published online: 1 June 2011

(C) The Author(s) 2011. This article is published with open access at Springerlink.com

\begin{abstract}
Abscisic acid, stress, ripening proteins (ASR) are a family of plant-specific small hydrophilic proteins. Studies in various plant species have highlighted their role in increased resistance to abiotic stress, including drought, but their specific function remains unknown. As a first step toward their potential use in crop improvement, we investigated the structure and regulation of the Asr gene family in Musa species (bananas and plantains). We determined that the Musa Asr gene family contained at least four members, all of which exhibited the typical two exons, one intron structure of Asr genes and the "ABA/WDS" (abscisic acid/ water deficit stress) domain characteristic of Asr genes.
\end{abstract}

Electronic supplementary material The online version of this article (doi:10.1007/s00425-011-1421-0) contains supplementary material, which is available to authorized users.

I. M. Henry · S. C. Carpentier - S. Pampurova .

A. Van Hoylandt $\cdot$ B. Panis $\cdot$ R. Swennen $\cdot$ S. Remy $(\bowtie)$

Division of Crop Biotechnics,

Laboratory of Tropical Crop Improvement,

K.U.Leuven, Kasteelpark Arenberg 13, Bus 2455,

3001 Leuven, Belgium

e-mail: serge.remy@biw.kuleuven.be

S. Pampurova

VIB Department of Molecular Microbiology,

Institute of Botany and Microbiology,

K.U.Leuven Laboratory of Molecular Cell Biology,

Kasteelpark Arenberg 31, Bus 2438, 3001 Leuven, Belgium

R. Swennen

Bioversity International, Kasteelpark Arenberg 13,

Bus 2455, 3001 Leuven, Belgium

Present Address:

I. M. Henry

Department of Plant Biology and Genome Center,

University of California Davis, 451 E. Health Sciences Drive,

Davis, CA 95616, USA
Phylogenetic analyses determined that the Musa Asr genes were closely related to each other, probably as the product of recent duplication events. For two of the four members, two versions corresponding to the two sub-genomes of Musa, acuminata and balbisiana were identified. Gene expression and protein analyses were performed and $A s r$ expression could be detected in meristem cultures, root, pseudostem, leaf and cormus. In meristem cultures, $m A s r l$ and $m A s r 3$ were induced by osmotic stress and wounding, while $m A s r 3$ and $m A s r 4$ were induced by exposure to ABA. mASR 3 exhibited the most variation both in terms of amino acid sequence and expression pattern, making it the most promising candidate for further functional study and use in crop improvement.

Keywords Abiotic stress - Abscisic acid · ASR . Musa . Osmotic stress

$\begin{array}{ll}\text { Abbreviations } \\ \text { ASR } & \text { Abscisic acid, stress, ripening } \\ \text { ABA } & \text { Abscisic acid } \\ \text { WDS } & \text { Water deficit stress } \\ \text { ITC } & \text { International Transit Center } \\ \text { BAC } & \text { Bacterial artificial chromosome } \\ \text { EST } & \text { Expressed sequence tag } \\ \text { q-RT-PCR } & \text { Quantitative real-time polymerase chain } \\ & \text { reaction } \\ \text { P4 } & \text { Proliferation medium } \\ \text { 2DE } & \text { Two-dimensional electrophoresis } \\ \text { ARP } & \text { ABA-responsive protein }\end{array}$

\section{Introduction}

Drought, high salinity and freezing all impose osmotic stress on plants and negatively affect their growth, 
development and/or productivity. The ability of each species to tolerate water deficit plays a critical role in determining its productivity and its natural geographical distribution. The effects of water deficit both on plant physiology and at the cellular level are highly complex (Ingram and Bartels 1996). Yet, transcriptome and proteome analyses using various model organisms have revealed some of the underlying molecular processes related to water stress (Seki et al. 2001; Rajjou et al. 2006; Shinozaki and Yamaguchi-Shinozaki 2007). Many of the genes involved in this response have been identified (Xiong and Zhu 2002), some of which respond to osmotic stress through abscisic acid (ABA) signaling. Among these, the abscisic acid, stress, ripening (ASR) protein has fostered growing interest as a potential key regulator of plant response to a variety of stresses, including osmotic stress (Cakir et al. 2003; Carrari et al. 2004; Frankel et al. 2007; Konrad and Bar-Zvi 2008; Carpentier et al. 2010a).

The first ASR protein was identified as a water/ABA stress-induced protein in tomato (Iusem et al. 1993). Other members were later identified in tomato, revealing the existence of a gene family (Rossi et al. 1996). ASR proteins are specific to plants and the number of documented members in the Asr gene family differs among plant species. For example, there are six known members in rice (Philippe et al. 2010), four in tomato (Frankel et al. 2006), four in pine (Chang et al. 1996) and as many as nine in maize (highest number of members described so far; Zivy and Coursol, INRA, France, personal communication), but none in A. thaliana (Carrari et al. 2004; Yang et al. 2005). Comparison of sequences from different species suggests that family members from a single species are more closely related to each other than to Asr members from other species (Carrari et al. 2004; Philippe et al. 2010), suggesting that they originate from late duplication events and that the sub-function of each of the family members might be species specific (Philippe et al. 2010).

ASR proteins have been repeatedly identified as involved in the response to different abiotic stresses, such as water deficit, salt stress, and cold or osmotic stress in many plants such as tomato, potato, maize or rice (AmitaiZeigerson et al. 1995; Gilad et al. 1997; Riccardi et al. 1998; Vaidyanathan et al. 1999; Maskin et al. 2001). Moreover, over-expression of the lily ASR in A. thaliana resulted in enhanced drought and salt resistance, confirming its role in stress response even in a system where it is not endogenously present (Yang et al. 2005).

ASR proteins are small hydrophilic proteins, the exact function of which remains unclear. They are characterized by the presence of the ABA/WDS (abscisic acid/water deficit stress) amino acid domain (PF02496) (Canel et al. 1995; Padmanabhan et al. 1997). Some ASR proteins also contain a zinc-dependent DNA-binding domain (Kalifa et al. 2004) and have been demonstrated to bind DNA both in a monomeric and a homodimeric form (Maskin et al. 2007). ASR proteins have been found in the cytosol, where they have been shown to exhibit chaperone-like activity, possibly stabilizing other cytosolic proteins against denaturation (Konrad and Bar-Zvi 2008). ASR proteins have also been reported to be localized in the nucleus in different cell types (Kalifa et al. 2004; Wang et al. 2005; Konrad and Bar-Zvi 2008), where they might play a regulatory role, possibly as transcription factors or as chaperones (Wang et al. 2005; Frankel et al. 2006; Konrad and Bar-Zvi 2008). Finally, the fact that the expression of most Asr genes is induced both by sugars and ABA has led to the hypothesis that it might serve as a link between sugar and ABA signaling, possibly through the regulation of hexose uptake (Cakir et al. 2003; Carrari et al. 2004; Frankel et al. 2007).

In the present report, we investigated the structure, diversity and regulation of the Asr gene family in Musa species (banana and plantains). As an important food crop, the yield of banana and plantains is economically important worldwide and crucial for the livelihood of many small farmers. Banana varieties differ widely in their ability to tolerate abiotic stresses, including drought. Most of the cultivated varieties are diploid or triploid and contain various relative proportions of the genomes of Musa acuminata (A genome) and Musa balbisiana (B genome). It is believed that varieties with a high proportion of the B genome are generally more resistant to abiotic stress (Thomas et al. 1998). This is supported by the fact that those varieties cover more northern and drier geographical regions (De Langhe and de Maret 1999; Perrier et al. 2009).

ASR proteins represent a promising candidate for Musa improvement. An ASR protein was initially identified in a proteomics screen of two varieties exhibiting contrasting tolerance to osmotic stress (Carpentier et al. 2007) and where a unique isoform was detected in a tolerant $\mathrm{ABB}$ variety. Asr sequences were also identified in a screen of DATAMUSA, an ensemble of Musa EST sequences, suggesting that ASR proteins can be expressed in all tissues tested (roots, peel green, male flowers and roots) (Santos et al. 2009). We recently discovered that the mASR 1 protein was significantly up-regulated by osmotic stress (Carpentier et al. 2010a). Furthermore, an Asr gene (Asrl according to our classification of the Musa Asr family members) was recently identified from a Musa sp. (the exact genomic group of the variety described as "Musa paradisiaca L. ABB group" is unclear to us, Liu et al. 2010). These authors argued that overexpression of MpASR resulted in increased drought tolerance in Arabidopsis thaliana. Although only two transgenic lines were characterized in this study, and no correlation 
between increased drought tolerance and level of mASR expression in their transgenic A. thaliana lines was presented (Liu et al. 2010), these results together with the aforementioned findings suggest that ASR proteins play an important role in banana stress response as well. Here, we report on the structure, phylogenetic position and regulation of the Asr genes of various Musa cultivars and wild accessions.

\section{Materials and methods}

Plant material

Various Musa spp. (Musaceae, Musa) were used in conducting this study. They correspond to wild varieties and cultivated varieties (cultivars), and each contain a specific combinations of the genomes of the two main species of Musa: Musa acuminata L. A. Colla and Musa balbisiana L. A. Colla. All plant materials were originally obtained from the International Transit Center (ITC), Kasteelpark Arenberg 13 bus 2455, 3001 Leuven, Belgium and each Musa sp. can be uniquely identified by its ITC number.

\section{Meristem culture}

Multiple shoot meristem cultures of 'Cachaco' (ITC.0643), 'Mbwazirume' (ITC.0084), balbisiana (ITC.0545), 'Populu' (ITC.0335), banksii (ITC.0466) and 'Williams' (ITC.0365) were initiated and maintained on P4 medium (MS medium supplemented with $100 \mu \mathrm{M}$ 6-benzylaminopurine and $1 \mu \mathrm{M}$ indole acetic acid) as previously described (Strosse et al. 2006).

For the experiment investigating natural variation in Asr expression in different cultivars (Fig. 4) and the experiment investigating tissue-specific expression of Asr in 'Williams' (Fig. 5a), meristems were harvested 6 days after the last subculture and flash frozen in liquid nitrogen. For the other experiments, only meristems from 'Cachaco' were used.

For the experiment investigating the effect of sucrose and wounding by cutting (Fig. 6a, b), meristems were divided into three groups. Following 1 week of growth on standard P4 medium, all groups were subcultured (i.e., differentiated tissue was cut and removed) on day 0 and placed back on their respective growth medium (not fresh). The cutting control group was harvested $24 \mathrm{~h}$ later. The "control" and "sucrose" groups were transferred to fresh standard P4 medium (0.09 M sucrose) or fresh P4 medium containing $0.4 \mathrm{M}$ sucrose, respectively, on day 4 and meristems were harvested 2 days later (day 6) and flash frozen in liquid nitrogen.

For the experiment investigating the effect of ABA (Fig. 6a, c), meristems were divided into two groups. Both groups were subcultured and transferred to fresh P4 medium on day 1 . On day 4, the "control" group was transferred to fresh $\mathrm{P} 4$ medium again, while the "ABA" group was transferred to fresh $\mathrm{P} 4$ medium containing $100 \mu \mathrm{M}$ ABA. On day 7, all meristems were harvested and flash frozen in liquid nitrogen.

Plant growth experiments in greenhouse conditions

For the experiment investigating tissue-specific expression of Asr (Fig. 5), plants were grown in pots in a greenhouse in which the photoperiod was extended to $12 \mathrm{~h}$ by artificial light when needed. The temperature reached 26 and $18^{\circ} \mathrm{C}$ in the day and night, respectively, while the relative humidity ranged between 70 and $90 \%$. For the experiment comparing $m A s r$ expression in leaves and meristem cultures from cultivar 'Williams' (Fig. 5a), leaf tissue was harvested from the fifth leaf of plants that were at least 6 months old. For the experiment investigating $m A s r$ expression in the first and second leaf of 'Cachaco' plants and in the roots (Fig. 5b), "Leaf 1" corresponds to the youngest leaf (cigar leaf), "Leaf 2" corresponds to the second youngest leaf and "Root" material was collected by pooling five of the widest primary roots. Plants had produced at least 7 leaves ( $\sim 2.5-3$ months old) at the time of harvest. All tissue was flash-frozen in liquid nitrogen immediately after harvest.

\section{Determination of the Musa ASR sequences}

Screen of genomic DNA using degenerate primers: genomic DNA from 'Cachaco' (ABB) was used for the initial gene discovery. Primers were designed based on Musa sequences from EST libraries and publicly available databases (see Table 1 for primer sequences). Using these primers, the complete sequence of mAsr 4 was obtained as well as fragments from the three other $m A s r$ sequences ranging between 100 and $400 \mathrm{bp}$.

Screening of BAC libraries: BAC libraries were obtained from the Musa Genomics Resource Center (MGRC, http:// Imcc.ieb.cz/dna-libraries/bananas) and screened to obtain the full-length genomic sequence of $m A s r 1,2$ and 3 . Genomic BAC libraries from 'Pisang Klutuk Wulung' (BB cultivar, MBP library) and 'Calcutta4' (AA cultivar, MA4 library) were screened for the presence of the desired Asr sequence. Briefly, 18 or 12 "superpools", for the MA4 and MBP libraries, respectively, were screened by PCR using degenerate primers designed from Asr sequences available in GenBank from Musa and other species. Positive superpools were screened further for identification of the positive BAC clone using the same PCR primers and conditions. Three positive clones, each harboring one of the mAsrl, $m A s r 2$ and $m A s r 3$ genes, were identified. Each of 
Table 1 List of primers used for the sequencing of the $m A s r$ genes and for determination of relative mRNA abundance using quantitative RT-PCR a EF1 $\alpha$ Elongation factor 1 alpha, ${ }^{\mathrm{b}}$ L2 ribosomal protein L2

\begin{tabular}{|c|c|c|}
\hline Application & Primer name & Primer sequence \\
\hline \multirow{12}{*}{$\begin{array}{c}\text { Quantitative RT-PCR } \\
\text { on } m A s r \text { sequences }\end{array}$} & mABA2_F & GCTTGCTACCTCTCGACCAC \\
\hline & mABA2_R & GTAGCTCCAGGCTTGCTGAC \\
\hline & mPGK_F & ATCATCGGAGGTGGTGACTC \\
\hline & mPGK_R & TTAGGCATCTTCAAGAGCAAG \\
\hline & qASR1_F & CGGTAGCAAACCACTGTTTCA \\
\hline & qASR1_R & GCTGTCTCGGAGTAGATCACC \\
\hline & qASR2_F & GTTTCACTTCGCAAGCAACA \\
\hline & qASR2_R & CTGGGGTTATAGGCGGTCTC \\
\hline & qASR3_F & CACCACСАССАССТСТТСТАА \\
\hline & qASR3_R & CATATGCACCAAACCAGCAC \\
\hline & qASR4_F & CTCCTCTGACAGTCCGTCAAT \\
\hline & qASR4_R & СТССТTCTTGGGGTCAACCT \\
\hline \multirow{12}{*}{$\begin{array}{c}\text { Quantitative RT-PCR } \\
\text { on reference genes }\end{array}$} & actin_F & GAGAAGATACAGTGTCTGGA \\
\hline & actin_R & ATTACCATCGAAATATTAAAAG \\
\hline & actin-11_F & CCCAAGGCAAACCGAGAGAAG \\
\hline & actin-11_R & GTGGCTCACACCATCACCAG \\
\hline & 25S r-DNA_F & ACATTGTCAGGTGGGGAGTT \\
\hline & 25S r-DNA_R & CCTTTTGTTCCACACGAGATT \\
\hline & tubulin_F & TGTTGCATCCTGGTACTGCT \\
\hline & tubulin_R & GGCTTTCTTGCACTGGTACAC \\
\hline & L2_F $F^{\mathrm{a}}$ & AGGGTTCATAGCCACACCAC \\
\hline & $\mathrm{L} 2 \_\mathrm{R}^{\mathrm{a}}$ & CCGAACTGAGAAGCCCCTAC \\
\hline & $\mathrm{EF} 1 \alpha \_F^{\mathrm{b}}$ & CGGAGCGTGAAAGAGGAAT \\
\hline & $\mathrm{EF} 1 \alpha \_\mathrm{R}^{\mathrm{b}}$ & ACCAGCTTCAAAACCACCAG \\
\hline \multirow{2}{*}{$\begin{array}{l}\text { Degenerate primers for } \\
\text { initial gene discovery }\end{array}$} & ASR_F & AGAARCACSAGGCSAARAAG \\
\hline & ASR_R & TCTTCTCRTGGTGCTCRTGG \\
\hline \multirow{10}{*}{$\begin{array}{l}\text { Sequencing primers for } \\
\text { phylogenetic analysis of } m A s r \text { genes }\end{array}$} & mASR1A_F & CGCAAACCACTGTTTCAGTCT \\
\hline & mASR1A_R & CAGCCAAGGCCATCATG \\
\hline & mASR1B_F & AGCAAACCACTGTTTCAGTTG \\
\hline & mASR1B_R & CAGCCAAGGCCATCACA \\
\hline & mASR2_F & GTTTCACTTCGCAAGCAACA \\
\hline & mASR2_R & AGCGCTTATTTGGGGGTAAT \\
\hline & mASR3_F & GCGGATGGCCGAGGAGAAGCATCACCG \\
\hline & mASR3_R & ATCCATGAACGTAAACACTCCACCTCAG \\
\hline & mASR4_F & CTCCTCTGACAGTCCGTCAAT \\
\hline & mASR4_R & CGTACGACAACCAGGAGATG \\
\hline
\end{tabular}

(GMGC, http://www.musagenomics.org) for 'Tani' (ITC. 1120) and 'Pisang Jari Buaya' (ITC.0312) or from newly extracted genomic DNA from 'Cachaco' (ITC.0643), 'Mbwazirume' (ITC.0084), 'Williams' (ITC.0365), 'Butuhan' (ITC.0565), banksii (ITC.0466), balbisiana (ITC.0545), 'Bluggoe' (ITC.1404), 'Pome' (ITC.0125), 'Pisang Awak (ITC.0339) and 'Orishele' (ITC.0517). PCR fragments were cloned and sequenced in both directions. The sequences obtained were cleaned of vector and primer sequences. Multiple sequences from the same cultivar or wild accession originate from independent cloning events. More clones per cultivar or wild accession were sequenced for the $m A s r 3$ gene than for the other three $m A s r$ genes, since it exhibited 
more variation within and between cultivars than the other three genes (see "Results"). Protein sequences were derived from genomic sequences after alignment of genomic sequences with cDNA sequence from at least one cultivar, removal of intron sequence and in silico translation. All $m A s r 1, m A s r 2$, partial $m A s r 3$ and $m A s r 4$ sequences obtained in the context of this study were deposited in Genbank under the following accession numbers: GU134740 to GU134760 for $m A s r 1, \mathrm{GU} 134761$ to GU134775 for $m A s r 2$, GU134776 to GU134782 for $m A s r 3$ and GU134735 to GU134739 for $m A s r 4$. Full-length $m A s r 3$ sequences can be found in Online Resource 4.

Multiple alignments of DNA or protein sequences were created using the ClustalX freeware, version 2.1.12 (Larkin et al. 2007). ClustalX 2.1.12 was also used to construct phylogenetic trees, using the neighbor-joining algorithm, with 1,000 replicate bootstrap tests. Trees were visualized using the njplot freeware (http://pbil.univ-lyon1.fr/ software/njplot.html). Sequences used for phylogenetic analysis either originated from this work or from publicly available databases (National Center for Biotechnology Information, http://www.ncbi.nih.gov). For the alignment depicted in Fig. 2, different sequences of the same gene and from the same cultivar or wild accession were kept separate if the nucleotide sequences differed by more than $1 \%$. If the nucleotide sequences differed by less than $1 \%$, only one sequence was retained (in the case of only two sequences) or a consensus sequence was derived (if there were three or more sequences). For $m A s r l$ to $m A s r 4$, a $1 \%$ error rate corresponds to a maximum of $4,5,5$ and $4 \mathrm{bp}$, respectively. A detailed description of the origin of each of the sequences used for this phylogenetic analysis can be found in Online Resource 1.

\section{Analysis of $m A S R$ gene expression}

Sample material was harvested as described above and stored at $-80^{\circ} \mathrm{C}$ until it was ground in liquid nitrogen for RNA extraction. Total RNA was extracted using the RNeasy plus mini kit (Qiagen Sciences), according to manufacturer's recommendations except for the addition of PVP40000 to the lysis buffer to a final concentration of $5 \mathrm{mg} / \mathrm{ml}$. Next, RNA was treated with DNaseI (Applied Biosystems/Ambion) for $45 \mathrm{~min}$ at $37^{\circ} \mathrm{C}$. Last, RNA was purified through a phenol-chloroform/IAA precipitation purification step. To verify the absence of residual genomic DNA, a real-time PCR was performed using DNase-treated RNA as template (see below). Only samples for which no amplification products could be detected after 40 cycles of PCR amplification were further processed. cDNA synthesis was performed on a known amount of RNA (1,000-3,000 ng, depending on the experiment) using the RevertAid $^{\mathrm{TM}} \mathrm{H}$ Minus First Strand cDNA Synthesis Kit
(Fermentas) and olido(dT) 18 primers, as recommended by the manufacturer.

Real-time RT-PCR was performed in a Corbett RotorGene 3000 (Qiagen). Reactions included: $12.5 \mu \mathrm{l} 2 \times$ Absolute SYBR ${ }^{\circledR}$ Green I master mix (Thermo Scientific, Epsom, United Kingdom), $150 \mathrm{nM}$ of reverse and forward primers (see Table 1 for sequences), $2 \mu$ of template (cDNA or gDNA or water) and water to reach a final volume of $25 \mu \mathrm{l}$. Each experiment included four to six biological replicates of each sample type and two technical replicates of all samples. For each independent experiment, all samples were run simultaneously. A set of six reference genes (actin, actin-11, 25S r-DNA, tubulin, elongation factor-1 $\alpha(\mathrm{EF} 1 \alpha)$ and ribosomal protein L2 (L2); see Table 1 for primer sequences) were run in addition to the four $m A s r$ genes. The most appropriate pair of reference genes was identified using geNorm v3.4, as previously described (Vandesompele et al. 2002) and used for normalization purposes. For comparison of cultivars (Fig. 4a), the two reference genes used for normalization were actin11 and tubulin. For comparison of tissue types in 'Williams' (Fig. 5a), the two reference genes used for normalization were EF1 $\alpha$ and $25 \mathrm{~S}$ r-DNA. For comparison of tissue types in 'Cachaco' (Fig. 5b), the two reference genes used for normalization were EF1 $\alpha$ and L2. For investigating the effect of exposure to high concentration of sucrose (Fig. 6b), the two reference genes used for normalization were EF1 $\alpha$ and actin11. For investigating the effect of exposure to ABA (Fig. 6c), the two reference genes used for normalization were actin11 and L2. Finally, relative mRNA concentrations were averaged over all biological replicates. After checking for normality and homogeneity of variance, means were compared on a pairwise basis using Student's $t$ tests and considered significantly different when the obtained $P$ value was $<0.05$.

For investigating the effect of high concentration of sucrose, the phosphoglycerate kinase $(P G K)$ encoding gene was used as a positive control since it had been previously demonstrated that the protein was more abundant in meristem cultures after induction with high concentrations of sucrose (Carpentier et al. 2007; Carpentier et al. 2010b). For investigating the effect of exposure to ABA, the $m A R P$ (ABA-responsive protein) gene was used as a positive control based on its homology to the A. thaliana "ABAresponsive protein-like" gene (At5g13200), known to be up-regulated by ABA exposure (Yazaki et al. 2004).

Analysis of protein abundance

2D-Gel electrophoresis (2DE) was performed via phenol extraction as previously described (Carpentier et al. 2005). For Western blots, small immobilized pH gradient (IPG) strips were used (7 cm pI 3_10 (GE Healthcare)). After 
2DE, proteins were blotted to a nitrocellulose membrane (GE Healthcare) via semi-dry transfer (Owl HEP-3 Semi Dry, Thermo Scientific) at $1 \mathrm{~mA} / \mathrm{cm}^{2}$ for $60 \mathrm{~min}$ (cathode buffer: $0.04 \mathrm{M} \varepsilon$-aminocaproic acid, $10 \%$ SDS, $20 \%$ methanol; protein transfer buffer: $48 \mathrm{mM}$ Tris, $39 \mathrm{mM}$ glycine, $0.037 \%$ SDS, $20 \%$ methanol, MQ $\mathrm{H}_{2} \mathrm{O}$; anode buffer I: $0.3 \mathrm{M}$ Tris, $20 \%$ methanol; anode buffer II: $0.025 \mathrm{M}$ Tris, $20 \%$ methanol). Membranes were blocked with 5\% skimmed milk powder in PBST $(20 \mathrm{mM}$ potassium phosphate, $150 \mathrm{mM} \mathrm{NaCl}, 0.5 \%$ (w/v) Tween-20, $\mathrm{pH}$ 7.2) for $60 \mathrm{~min}$. After three washes in PBST, the membranes were incubated with primary antibody $(1 / 2,000$ in PBST) [rabbit anti-ASR polyclonal antibody was raised against a custom peptide matching the sequence ETAYSGGGDYASGYT (Eurogentec, Belgium)] overnight at $4^{\circ} \mathrm{C}$. After washing in PBST, the membranes were incubated with secondary antibody goat anti-rabbit (GE Healthcare) $(1 / 2,500$ in PBST) for $60 \mathrm{~min}$. Visualization of the proteins was realized with AEC (3-amino-9-ethylcarbazole), according to the manufacturer's recommendations (Sigma, Belgium).

\section{Results}

What is the structure of the $m A s r$ gene family?

Through a combination of degenerate PCR, EST library screening and BAC sequencing, we discovered four Asr genes in banana. These genes have been named $m A s r l$ through mAsr4 (musa Asr). A total of more than 20,000 expressed sequence tags (EST) from cDNA libraries constructed from different tissue types or from samples subjected to various stresses (Santos et al. 2005; Roux et al. 2008; Carpentier et al. 2010a) were screened for additional banana Asr sequences.

All four genes contain two exons separated by a single intron, as do other Asr sequences from other species (Fig. 1). In banana, the mASR4 protein is shorter than the other three members of the family (103 vs. 144-146 amino acids, respectively). All four mASR proteins also contain the ABA/WDS domain, characteristic of the ASR family (PFAM domain PF02496).

What is the phylogenetic relationship between the four mASR proteins?

Once all four members of the mASR family were identified, each member was sequenced from different cultivars and wild varieties of Musa. Cultivars and wild varieties were chosen such that both diploid and triploid cultivars as well as most possible combinations of A and B genomes were represented. In total, we analyzed $18 \mathrm{mAsrl}$ sequences from 13 cultivars, 11 mAsr 2 sequences from 7

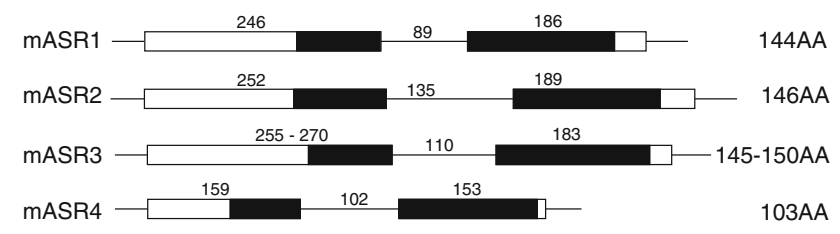

Fig. 1 Structure of the $m A s r$ gene family. Intron-exon structure of the $4 \mathrm{mAsr}$ genes from Musa. Each member contains two exons (boxes) separated by one short intron (line). The parts of the exons containing the ABA/WDS domain is highlighted in black. Numbers above the exons and introns indicate the length of these elements (bp). The length of the first exon in $m A s r 3$ varies between 255 and 270 bp depending on the sequence

cultivars, $9 \mathrm{mAsr} 3$ sequences from 5 cultivars and $4 \mathrm{mAsr} 4$ sequences from 4 cultivars. All genomic sequences were aligned (see "Materials and methods" for details) and a phylogenetic tree was constructed (Fig. 2). As expected from their size, mAsr4 sequences were most divergent, while the other three members of the family were relatively close to each other.

We originally focused on mASR1 because it was one of the most highly expressed proteins in our meristem cultures (Carpentier et al. 2007). The mAsrl sequences obtained clearly clustered into two groups. The sequences present in each group and the genotype of the cultivars from which they originated was consistent with one cluster containing alleles from the A genome and the other containing alleles from the B genome (Fig. 2, gray box). Amino acid sequences from 13 Musa varieties (wild varieties and cultivated varieties) were aligned, and sequences in the A and B groups consistently differed by only two amino acids (Online Resource 3). Yet, on a 2DE gel, the A and the B isoforms were clearly separated (Carpentier et al. 2010b) (Fig. 4b).

Fewer sequences were obtained from $m A s r 2$. Alignment of the genomic sequences, including the intron, allowed for the identification of two clusters, potentially corresponding to the A and B genomes (data not shown). Translation analysis of these sequences revealed no difference specific to the A and B version at the amino acid level. The cluster of $m A s r 3$ genomic sequences also contained a sub-cluster (Fig. 2, in gray) that could correspond to the B version of this gene. Sequence variability in the $m A s r 3$ genes and mASR 3 proteins was much more pronounced than in the other members of the mASR family (Online Resource 4 and Table 2). Finally, the number of different genomic sequences obtained from mAsr4 was too limited (4, Table 2) to be conclusive as to the difference between the $\mathrm{A}$ and $\mathrm{B}$ versions of this gene.

Next, we investigated the position of the mASRs with respect to ASR sequences from other species. We included sequences from both the $\mathrm{A}$ and the B genomes of Musa: 


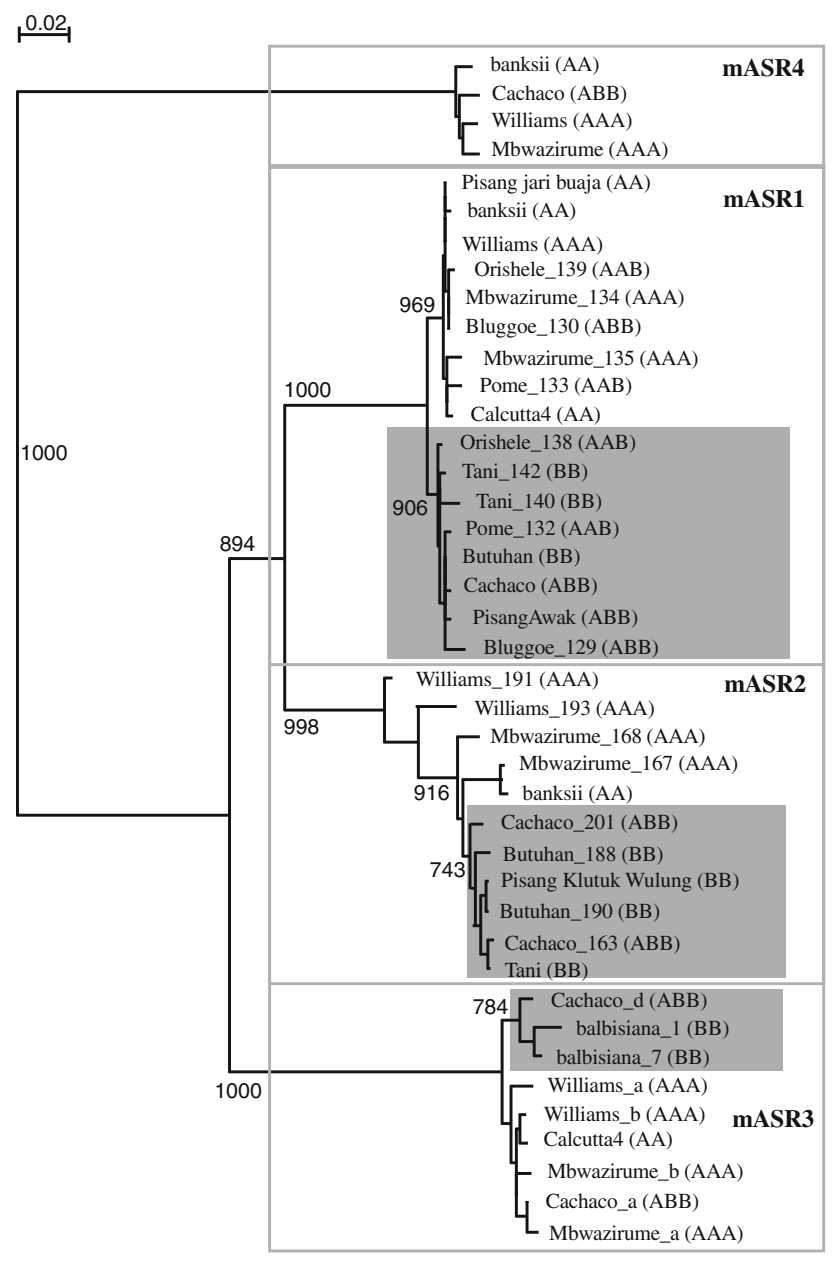

Fig. 2 Phylogenetic relationship between $m A s r$ genomic sequences in various wild and cultivated accessions of Musa. Full-length genomic sequences (including the intron) were aligned using ClustalX and a neighbor-joining tree was constructed (bootstrap of 1,000). The distance between sequences is indicated by branch length. Bootstrap values between major groups and $>75 \%$ are indicated. Gray boxes indicate putative sub-clusters associated with the $\mathrm{A}$ and $\mathrm{B}$ genomes for $m A s r 1, m A s r 2$ and $m A s r 3$. For each sequence, the name of the cultivar or wild variety is indicated and the genome composition in brackets. Sequences from the same cultivar or wild variety are distinguished by the name of the clone corresponding to each particular sequence. The gene ID of each of the genes used for the construction of this tree can be found in Online Resource 1

from 'Williams' (AAA), 'Tani' (BB) and/or balbisiana (wild $\mathrm{BB}$ ) from the four mASR proteins and aligned them to previously identified ASR sequences from other species (Fig. 3). All four mASRs protein clustered together rather than with ASR proteins from other species, suggesting that they were the product of recent duplication events rather than orthologs of Asr genes found in other species. Interestingly, the relationship between mASR1, mASR2 and mASR3 was different when using protein sequences rather than genomic sequences (Figs. 2, 3). Specifically, the protein sequences of $\mathrm{mAsr} 1$ clustered with those of $\mathrm{mAsr} 3$, while genomic sequences of $\mathrm{mAsr} 1$ and $\mathrm{mAsr} 2$ clustered
Table 2 Percentage of identity between mASR proteins from different cultivars or wild accessions

\begin{tabular}{lllr}
\hline Protein & From & To & $\mathrm{N}^{\mathrm{a}}$ \\
\hline mASR1 & 92.9 & 99.3 & 11 \\
mASR2 & 95.9 & 99.3 & 8 \\
mASR3 & 83.9 & 98.6 & 9 \\
mASR4 & 99.0 & $\mathrm{NA}^{\mathrm{b}}$ & 2 \\
\hline
\end{tabular}

${ }^{a}$ Number of different sequences available

b Only two sequences available

together. This relationship held true and was well supported when all mASR protein sequences obtained in the context of this study were aligned (data not shown).

Why did we not obtain an A version of $m A s r l$ from Cachaco?

Surprisingly, the cultivar from which ASR was originally identified, 'Cachaco', is of the ABB genomic composition, but no A version of $m A s r l$ could be found in the proteomics study (Fig. 4b), in the Musa cDNA libraries (Carpentier et al. 2010a) or when using PCR primers specific for the A version of $m A s r l$ at the gDNA level. Additionally, sequencing fragments that were amplified using primers that match perfectly to both the A and B version of Asrl resulted in no ambiguous base at the positions where $m A s r 1 A$ and B differ, while ambiguity was readily apparently in similar sequences obtained from other hybrid cultivars (data not shown). These results suggest that the $m A s r l A$ transcript as well as its encoding gene are lacking in 'Cachaco'. Further proteome analysis (2DE separation and de novo sequencing (Carpentier et al. 2010b) has revealed that 'Cachaco' does contain typical A genotype isoforms of other proteins. These data demonstrate that 'Cachaco' does indeed carry an A genome and that the A version of Asr was lost. A similar situation has been observed for other proteins in various cultivars (De Langhe et al. 2010). In the case of $m A s r l$, whether or not this is the result of positive selection for the $\mathrm{B}$ isoform remains to be determined.

Is there natural variation for mASR expression?

In the abovementioned proteomics screen investigating the effect of osmotic stress on gene regulation in banana meristems (Carpentier et al. 2007), ASR1 was identified as one of the most abundant proteins in meristem cultures of 'Cachaco' (ABB), a cultivar exhibiting enhanced resistance to osmotic stress in meristem cultures. In 'Cachaco', ASR4 protein could be detected as well, but weakly. Furthermore, the screening of an EST library containing over 10,000 transcripts from banana meristems grown under osmotic stress (Carpentier et al. 2010a) suggested also that mAsrl 


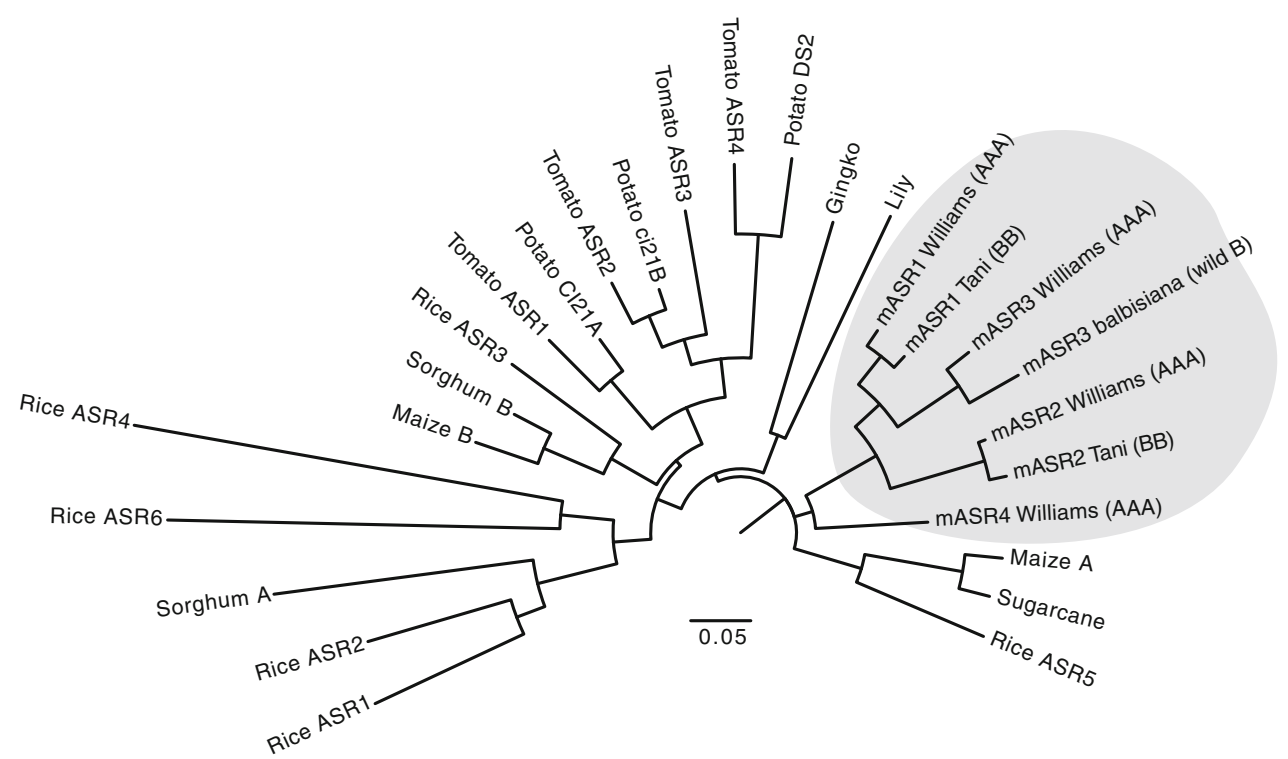

Fig. 3 Neighbor-joining tree of ASR protein sequences from various plants species. Full-length protein sequences were aligned using ClustalX and an unrooted neighbor-joining tree was constructed (bootstrap of 1,000). The distance between sequences is indicated by branch length. The four members of the $m A s r$ family are enclosed in the gray box and represented by sequences from 'Williams' (AAA, mASR1_clone173, mASR2_clone193, mASR3_clone_a and mASR4_clone170), 'Tani' (BB, mASR1_clone140, mASR2_ clone197) and balbisiana (BB, mASR3 clone1). Sequences from other plant species are available in NCBI: NP_001152333.1 (Maize B), XP_002447825.1 (Sorghum B), XP_002457802.1 (Sorghum A),

was also one of the most abundant transcripts ( 73 hits), while $m A s r 2,3$ and 4 were either not present or only rarely found (1 hit, no hit and 3 hits, respectively).

To further document $m A s r$ regulation, primers specific to each family member, but amplifying both the A and B version of each gene, were designed for expression analysis using quantitative real-time PCR (q-RT-PCR). Gene expression could be detected from all four $m A s r$ genes in non-stressed meristem cultures of 'Cachaco' (ABB cultivar), demonstrating that at least one allele per $m A s r$ gene is functional (Fig. 4).

Next, the expression level of the mAsr genes was investigated in other cultivars. mRNA abundance was compared between meristem cultures of 'Cachaco' and two AAA cultivars, 'Williams' and 'Mbwazirume' (Fig. 4a). All four $m A s r$ genes were expressed in all three cultivars, but variation in relative expression levels could be identified. Specifically, the expression of all $m A s r$ genes was significantly higher in 'Mbwazirume' than in 'Cachaco', especially for $m A s r 2$ ( $>90$-fold increase) and $m A s r 3$ ( $\sim$ eightfold increase). $m A s r$ expression was not significantly different in 'Williams' than in 'Cachaco' except for $m A s r l$, which exhibited slightly lower expression in 'Williams' than in 'Cachaco' (<twofold).
AAT57940.1 (Sugarcane), CAA72998.1 (Maize A), ACZ50736 (mASR4), ACZ60133.1 (mASR1), ACZ60138.1 (mASR1), ACZ 50744.1 (mASR2), ACZ50739.1 (mASR2), EEC72202 (Rice ASR2), NP_001045487 (Rice ASR6), BAD28236 (Rice ASR3), NP 001173936 (Rice ASR4), NP_001065841 (Rice ASR5), NP_ 001045459 (Rice ASR1), 2282019 (Tomato ASR1), 584787 (Tomato ASR2), 400471 (Tomato ASR3), AAY98032 (Tomato ASR4), 4098248 (Potato CI21A), 4098250 (Potato ci21B), 23095773 (Potato DS2), 6525055 (Lily) and 38532363 (Gingko). The nomenclature of the Rice ASR proteins comes from (Philippe et al. 2010)

Finally, ASR 1 protein could be detected in all cultivars tested using two-dimensional (2D) gel electrophoresis as well (Fig. 4b). The A and B versions of the proteins were located in different spots, because two amino acids are consistently different between the A and B versions of the protein (Online Resource 3), resulting in a significant shift in isoelectric point. This is illustrated by the position of the mASR 1 spot in various cultivars and the presence of two distinct spots in the AAB cultivar 'Populu' (Fig. 4b). As discussed above, only one spot could be detected in the 'Cachaco' (ABB) cultivar, due to the absence of the mAsrlA gene in the 'Cachaco' genome. The position of the ASR proteins identified on the 2D gels was verified by Western blotting using an anti-ASR 1 peptide polyclonal antibody (data not shown).

Taken together, these results demonstrate that all four $m A s r$ genes are active and that $m A s r l$ and $m A s r 4$ are the most abundantly expressed, but the level of expression of $m A s r 2$ and $m A s r 3$ exhibited wide variations depending on the cultivar.

What is the pattern of expression of the $m A s r$ genes?

To compare relative mRNA abundance in different tissues, q-RT-PCR was performed simultaneously on different tissues from the same cultivar (Fig. 5). First, mRNA 


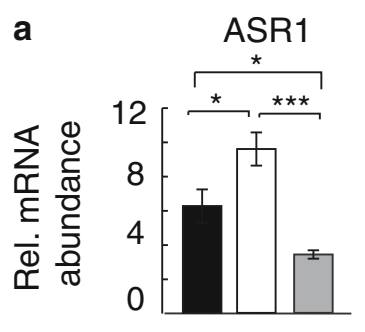

Cachaco (ABB)

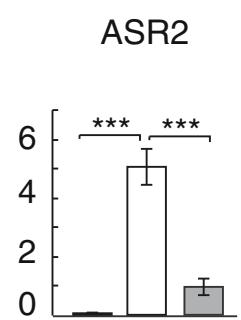

Mbwazirume (AAA)
ASR3

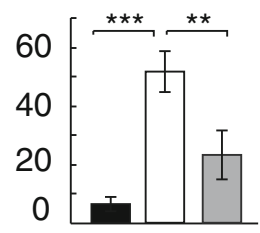

ASR4

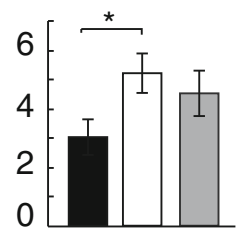

Williams (AAA)

b

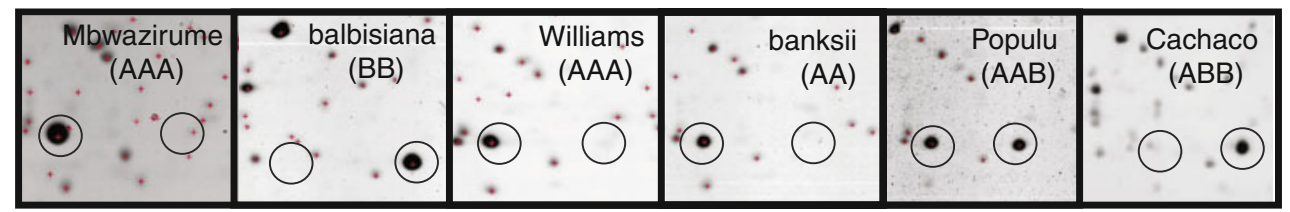

Fig. 4 Natural variation for $m A s r$ expression. a Relative mRNA abundance of mAsrl to mAsr4 in meristem cultures from three different cultivars. mRNA abundance was measured by quantitative RT-PCR (see "Materials and methods" for details). The two reference genes used for normalization were actin11 and tubulin. mRNA abundance was measured in 'Cachaco' (black bars), 'Mbwaziume' (white bars) and 'Williams' (gray bars) meristem cultures. Standard errors are represented. For each member of the $m A s r$ family, mean relative abundance values were compared to each other on a pair-wise basis using Student's $t$ tests. Comparisons that were significant are indicated by one, two or three asterisks depending

abundance was compared between meristem cultures and greenhouse leaves of 'Williams' (Fig. 5a). The relative mRNA abundance of the $m A s r$ genes was tissue specific. All $m A s r$ genes exhibited high expression in leaves, except for $m A s r 4$, which exhibited higher expression levels in meristems (Fig. 5a). The difference was particularly noticeable for $m A s r 2$ and $m A s r 3$ ( $\sim$ tenfold higher expression levels in leaves than in meristems). Similarly, mRNA abundance was compared in tissues from the first leaf (cigar leaf or last unrolled leaf), second leaf and roots of the 'Cachaco' cultivar (Fig. 5b). Again, all $m A s r$ members were expressed in all tissue types tested, but at different levels and each gene exhibited a specific pattern of expression (Fig. 5b). The level of expression was significantly different in roots compared to the second leaf (all four mAsr members) and first leaf (mAsr4). Specifically, $m A s r 1$ and $m A s r 2$ expression was lower in roots than in the second leaf, whereas the opposite was observed for $m A s r 3$ and $m A s r 4$.

Finally, 2DE Western-blot analysis of ASR proteins was performed on tissue from roots, leaves, pseudostem and corm tissue from 'Cachaco' and confirmed that the ASR1 protein was present in all of these tissues (Online Resource 2).

Are the $m A s r$ genes regulated by sugar and ABA?

Asr genes from various species have been demonstrated to be regulated by sugar and/or ABA (Rossi et al. 1998; Vaidyanathan et al. 1999). We tested whether this was true of on whether the $P$ value was $<0.05,<0.01$ or $<0.001$, respectively. b Two-dimensional gel of proteins extracted from meristem cultures of different Musa cultivars or wild accessions. Only the relevant portion of the gels, including the positions of the mASR1A (on the left) and mASR1B (on the right) proteins, is shown (Mw $\sim 22 \mathrm{kDa}$ and $\mathrm{pI} \sim 6.30$ or 6.50 for the $\mathrm{A}$ or $\mathrm{B}$ versions of the protein, respectively). These positions are indicated by circles around the protein spots where the protein is present and empty circles where the protein is not detectable. A full gel image for 'Cachaco' meristems can be found at http://www.pdata.ua.ac.be/musa

the $m A s r$ genes as well. mAsr expression was compared in 'Cachaco' meristems cultured on regular medium or on growth medium supplemented with $0.4 \mathrm{M}$ sucrose. Additionally, the effect of the wounding stress associated with subculturing was also assessed (see "Materials and methods" for details). Relative mRNA abundance was compared between the three experimental treatments (Fig. 6a). As expected, the positive control $(P G K)$ exhibited increased expression in sucrose-treated samples, while cutting did not affect its expression. We could show that the expression levels of both $m A s r l$ and $m A s r 3$ were significantly increased by both high sucrose concentration and wounding by cutting (Fig. 6b).

Finally, a similar experiment was conducted comparing relative mRNA abundance in 'Cachaco' meristems grown on regular medium and samples grown for 3 days on medium supplemented with $100 \mu \mathrm{M}$ ABA. As expected, expression of the positive control ( $m A R P$ ) was significantly induced by ABA exposure (Fig. 6c). We could show that the expression of $m A s r 3$ and $m A s r 4$ increased significantly in the presence of ABA ( $\sim 4$-fold and $\sim 2$-fold, respectively), but to lower extents than that of mARP ( $\sim 12$-fold).

\section{Discussion}

Asr genes encode small proteins with potentially important functions in response to a variety of stresses. This study 

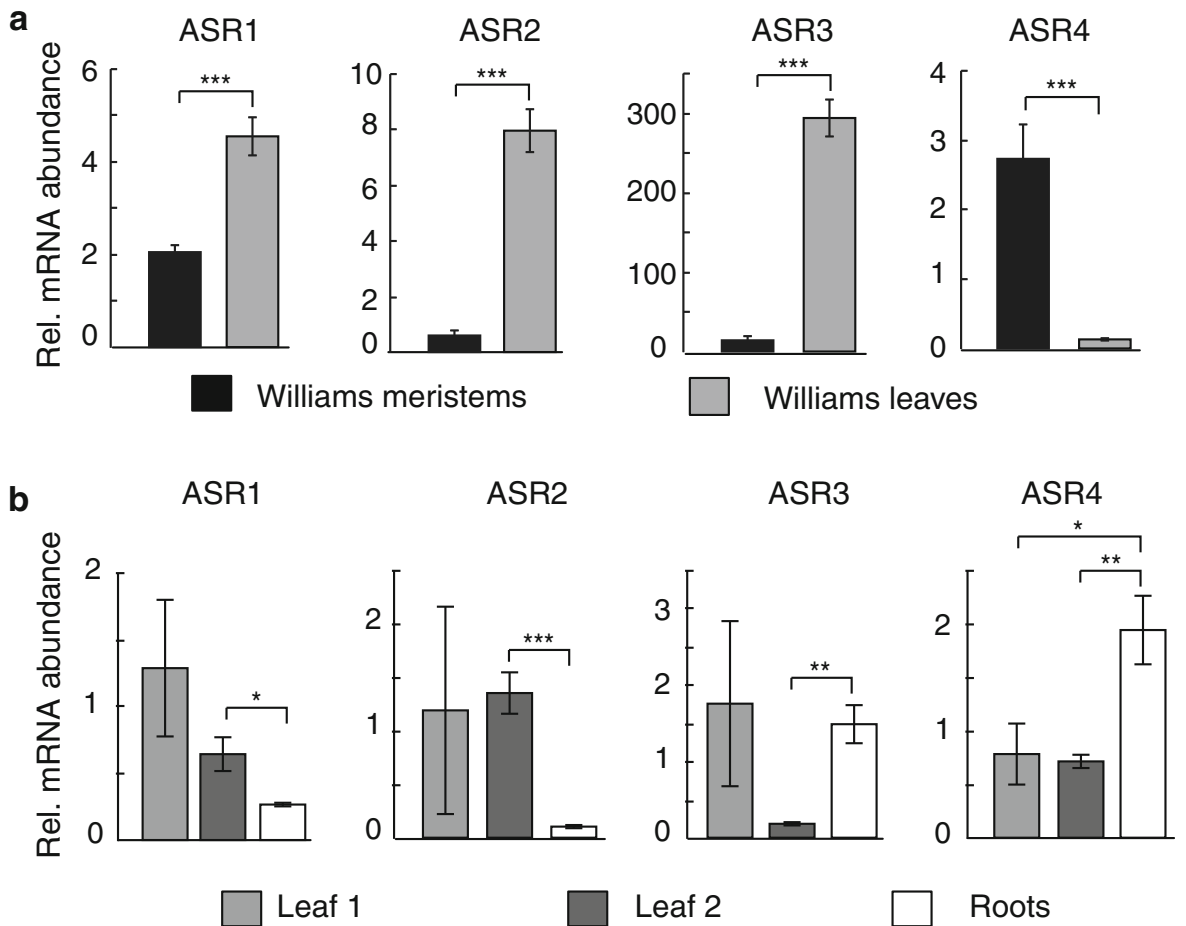

Fig. 5 Developmental and tissue-specific expression of the $m A s r$ family members. Relative mRNA abundance of $m A s r l$ to $m A s r 4$ was measured by quantitative RT-PCR. For each member of the $m A s r$ family, mean relative abundance values were calculated and compared to each other on a pair-wise basis using Student's $t$ tests. Standard errors are represented. Comparisons that were significant are indicated by one, two or three asterisks depending on whether the $P$ value was $<0.05,<0.01$ or $<0.001$, respectively. a Comparison of mRNA abundance in greenhouse leaves and meristem cultures of the

aimed at characterizing the Asr gene family in Musa and to potentially identify promising candidates for crop improvement. We have identified 4 Asr genes from Musa, which all carry the ABA/WDS motif and the gene structure characteristic of Asr genes (Fig. 1). Through detailed analysis of the sequences of the $m A s r$ genes (Figs. 2,3) and documentation of their expression patterns (Figs. 4, 5, 6), we now have a detailed view of the specificities of each member of the family.

Origin of the four $m A s r s$

Banana is a monocotyledon, but only distantly related to the more well-studied model system of rice (Aert et al. 2004; Cheung and Town 2007), providing a different angle to investigate the evolution of the Asr gene family. Indeed, the four $m A s r$ genes are phylogenetically close to each other, with respect to other Asr genes from other species including those of other monocots. This situation is similar to that observed in some species or group of species. For example, the four known Asr genes from pine all cluster together and away from Asr genes from other species, while Asr genes from tomato and potato all cluster together

'Williams' (AAA) cultivar. The two reference genes used for normalization were EF1 $\alpha$ and $25 \mathrm{~S}$ r-DNA. Values obtained for leaves (gray bars) are expressed relative to values obtained for meristem cultures (black bars). b Comparison of mRNA abundance in the first (light gray bars) and second leaves (dark gray bars) as well as root tissue (white bars) from greenhouse plants of the 'Cachaco' (ABB) cultivar. The two reference genes used for normalization were EF1 $\alpha$ and $\mathrm{L} 2$

with an internal pair-wise organization of potato and tomato orthologs (Frankel et al. 2006). Interestingly, in tomato, all four Asr genes are located next to each other on chromosome IV (Frankel et al. 2006), suggestive of tandem duplications specific to Asr or a small portion of the genome containing Asr. In rice, on the other hand, the six Asr genes are located on four different chromosomes (Philippe et al. 2010). The mechanisms leading to the appearance of the different members of Asr families might thus be species dependent as well.

In banana, three of the four members cluster very closely together, while the fourth $(m A s r 4)$ clusters more distantly (Figs. 2, 3; Table 2). Possibly, one or several of the $m A s r$ homologs originated from whole-genome duplication events. Indeed, a recent study of Musa BAC sequences suggested a whole genome duplication event specific to Musa species and estimated to have happened approximately 61 million years ago (Lescot et al. 2008). Assuredly, the sequence of the Musa genome (currently underway) will help shed light on the mechanisms underlying the evolution of the $m A s r$ family in this genus. For example, it will indicate whether some of the $m A s r$ genes 

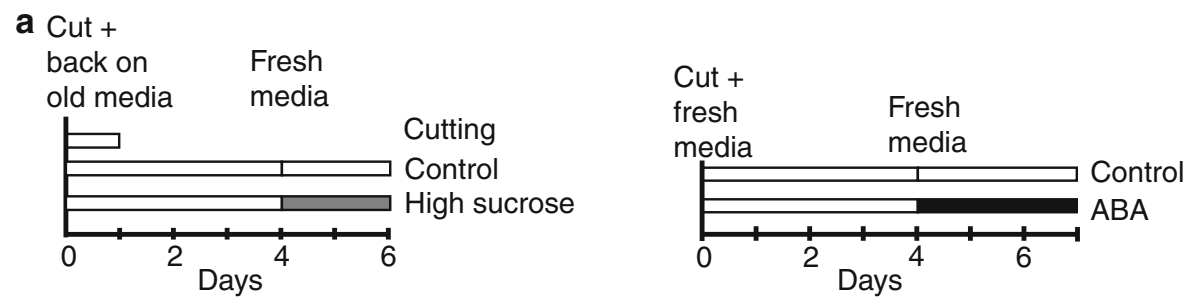

$\square$ Control P4 (0.09 M sucrose) $\square$ P4 + sucrose (0.4 M sucrose) $\square$ P4 + $100 \mu$ M ABA
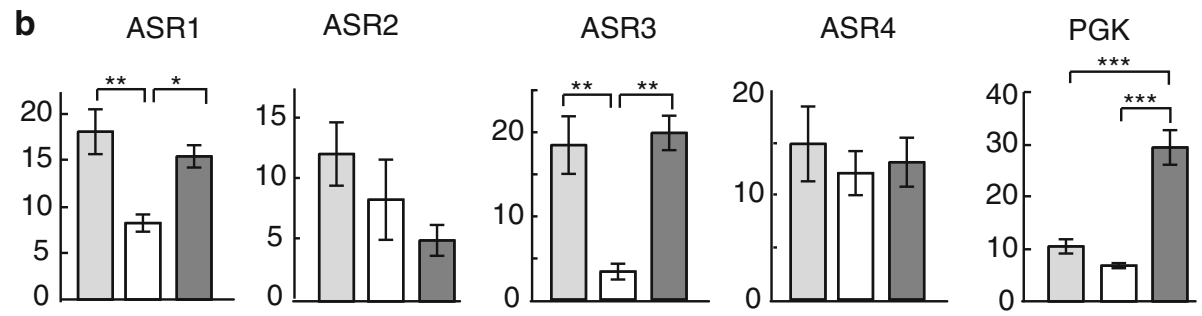

Control

High sucrose treatment

Cutting treatment

C

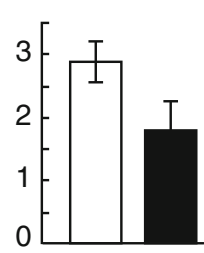

ASR2

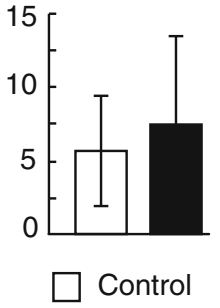

ASR3

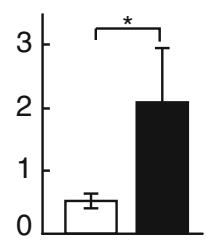

ASR4

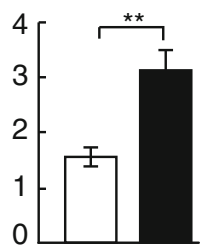

mARP

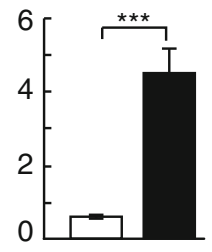

Fig. 6 Regulation of the $m A s r$ members following exposure to sucrose, wounding (cutting) stress or ABA. The relative mRNA abundance of $m A s r l$ to $m A s r 4$ was measured by quantitative RT-PCR on meristem cultures of the 'Cachaco' cultivar (ABB). For each member of the $m A s r$ family, mean relative abundance values were compared to each other on a pair-wise basis using Student's $t$ tests. Standard errors are represented. Comparisons that were significant are indicated by one, two or three asterisks depending on whether the $P$ value was $<0.05,<0.01$ or $<0.001$, respectively. a Schematic view of the treatments applied to each of the sample types. For the "sucrose experiment", samples were cut on day 0 and put back on their respective standard growth media. The "cut" samples were harvested the next day. The other two types of samples were transferred to fresh medium (either standard growth medium or standard growth medium supplemented with $0.4 \mathrm{M}$ sucrose) on day 4 and harvested 2 days later. For the "ABA experiment", samples were cut on day 0 and placed on fresh growth media. On day 4, they were placed on either fresh standard growth medium or fresh standard growth medium supplemented with $100 \mu \mathrm{M}$ ABA. Samples were harvested 3 days later. b Comparison of mRNA abundance in the different sample types illustrated in a: control (white bars), cut (light gray bars) and high sucrose samples (dark gray bars). The two reference genes used for normalization were EF1 $\alpha$ and actin11. The expression of phosphoglycerate kinase (mPGK) was used as a positive control c Comparison of mRNA abundance in meristems grown on standard growth medium (white bars) or grown on standard growth medium supplemented with $100 \mu \mathrm{M}$ ABA (black bars) for 3 days before harvest. The two reference genes used for normalization were actin11 and L2. The expression of an ABA-responsive protein (mARP) was used a positive control are located on the same chromosome, suggestive of tandem duplication or whether another large-scale duplication event can be detected in the genome.

$m A s r$ genes are ubiquitously expressed

We have demonstrated that at least one allele of each of the $m A s r$ genes was expressed in all tissues sampled (roots, leaves, meristems, pseudostem, corm). Moreover, the expression level of the different members varied depending on the cultivar, the developmental stage (for example, young versus older leaves) or the tissue sampled. However, we cannot rule out the possibility that the observed variation is due to preferential or exclusive PCR amplification from a subset of the alleles in certain cultivars, since we used only one primer set per gene.

This type of observation could be consistent with subfunctionalization and has also been observed in other species. For example, in tomato, Asrl's expression pattern exhibits very little variation, while the other members exhibit much more variable expression levels (Frankel 
et al. 2006): they exhibit variable responses to water-deficit stress and variable expression levels in different tissues (Maskin et al. 2008). Similarly, in banana meristems, mAsrl was one of the most abundant proteins detected by 2D gel electrophoresis, while mAsr4 was detected at a much lower level (Carpentier et al. 2007) and $m A s r 2$ and $m A s r 3$ were undetected at the protein level.

Both the hypothesized role of ASR as a protein chaperone in the cytoplasm (Wang et al. 2005; Frankel et al. 2006; Konrad and Bar-Zvi 2008) or DNA chaperone in the nucleus could be consistent with our findings. The sequence of mASR4 is most different from that of the three other mASRs. Most strikingly, it is much shorter (103 vs. 144-150 for the other three mASR proteins). A similar situation has been observed in tomato, where ASR1, ASR2 and ASR3 are shorter (between 108 and 114 AAs), while ASR4 is much longer (297 AAs). Frankel et al. (2006) hypothesized that the extra domain encodes an additional biochemical function. Finally, ASR proteins have also been categorized as class seven of the late embryogenesis abundant (LEA) proteins and five domains were identified within ASR sequences from various species (Battaglia et al. 2008). Domains 1, 2 and 3 are highly conserved, while domains 4 and 5 are only found in a subset of ASR proteins. Interestingly, all five domains are also found in mASR1, mASR2 and mASR3, while mASR4 lacks domain 5 and carries a heavily modified version of the middle domain (domain 1). It is thus possible that mASR4 performs a different or a subset of the function(s) performed by the other three members of the $m A s r$ gene family.

mAsr3 exhibits the most variation

In our survey of the response of $m A s r$ to abiotic stress, we have observed that $m A s r 3$ expression was low in control samples, but most induced by high sucrose, wounding stress or ABA treatment. An in-depth analysis of the promoter sequence of $m A s r 3$ would be instrumental to obtain more information about its potential role in stress response.

mAsr3 also exhibited the most sequence variation among the cultivars tested. Specifically, the sequences that formed the putative "B" cluster exhibited the highest variation of all sequences identified within the context of this work. Similar sequence variation has been observed for some members of Asr families from other species. In tomatoes, phylogenetic analysis of Asr2 genomic sequences from different varieties demonstrated accelerated positive selection correlated with colonization of dry environments (Frankel et al. 2003; Giombini et al. 2009). Similarly, an in-depth study of the rice Asr genes determined that OsAsr3 was the most divergent of the rice Asr genes and exhibited overall neutral selection at the species level, but directional selection in the sub-group of japonica found in tropical regions (Philippe et al. 2010). Musa balbisiana varieties are thought to be more drought tolerant than cultivars containing only A (from Musa acuminata) genomes (Nelson et al. 2006). Obtaining more sequences from mAsr3, especially from selected drought-tolerant and drought-sensitive varieties, will be instrumental in determining whether such a situation also occurs in Musa.

\section{Supplementary materials}

All sequences obtained in the context of this study have either been submitted to the nucleotide database at NCBI (National Center for Biotechnology Information, http:// www.ncbi.nih.gov) or are available in Online Resource 4.

Acknowledgments We thank Dr. Jaroslav Dolezel and Dr. Eva Hribova (Institute for Experimental Biology, Olomouc, Czech republic) for their work on the ITS region of Cachaco. We thank the Global Musa Genomics Consortium (GMGC) for providing us with BAC libraries and BAC clones as well as the genomic DNA collection. We thank László Sági for providing genomic DNA from various cultivars, and Els Thiry and An Krauss for technical help. This work was supported by the Fonds Wetenschappelijk Onderzoek-Vlaanderen (Grant G029606N and postdoc fellowship of S.C.C.), the KUL Bijzonder Onderzoek Fonds (to IMH, postdoctoral fellowship and to SP, predoctoral fellowship) and KUL Industriëel Onderzoek Fonds (Grant KP/08/001).

Open Access This article is distributed under the terms of the Creative Commons Attribution Noncommercial License which permits any noncommercial use, distribution, and reproduction in any medium, provided the original author(s) and source are credited.

\section{References}

Aert R, Sagi L, Volckaert G (2004) Gene content and density in banana (Musa acuminata) as revealed by genomic sequencing of BAC clones. Theor Appl Genet 109:129-139

Amitai-Zeigerson H, Scolnik PA, Bar-Zvi D (1995) Tomato Asrl mRNA and protein are transiently expressed following salt stress, osmotic stress and treatment with abscisic acid. Plant Sci 110:205-213

Battaglia M, Olvera-Carrillo Y, Garciarrubio A, Campos F, Covarrubias AA (2008) The enigmatic LEA proteins and other hydrophilins. Plant Physiol 148:6-24

Cakir B, Agasse A, Gaillard C, Saumonneau A, Delrot S, Atanassova $\mathrm{R}$ (2003) A grape ASR protein involved in sugar and abscisic acid signaling. Plant Cell 15:2165-2180

Canel C, Bailey-Serres JN, Roose ML (1995) Pummelo fruit transcript homologous to ripening-induced genes. Plant Physiol 108:1323-1324

Carpentier SC, Witters E, Laukens K, Deckers P, Swennen R, Panis B (2005) Preparation of protein extracts from recalcitrant plant tissues: an evaluation of different methods for two-dimensional gel electrophoresis analysis. Proteomics 5:2497-2507

Carpentier SC, Witters E, Laukens K, Van Onckelen H, Swennen R, Panis B (2007) Banana (Musa spp.) as a model to study the 
meristem proteome: acclimation to osmotic stress. Proteomics 7:92-105

Carpentier SC, Vertommen A, Swennen R, Witters E, Fortes C, Souza MT, Panis B (2010a) Sugar-mediated acclimation: The importance of sucrose metabolism in meristems. J Proteome Res 9:5038-5046

Carpentier SC, Panis B, Renaut J, Samyn B, Vertommen A, Vanhove A-C, Swennen R, Sergeant K (2010b) The use of 2D-electrophoresis and de novo sequencing to characterize inter- and intracultivar protein polymorphisms in an allopolyploid crop. doi: 10.1016/j.phytochem.2010.10.016

Carrari F, Fernie AR, Iusem ND (2004) Heard it through the grapevine? ABA and sugar cross-talk: the ASR story. Trends Plant Sci 9:57-59

Chang S, Puryear JD, Dias MADL, Funkhouser EA, Newton RJ, Cairney J (1996) Gene expression under water deficit in loblolly pine (Pinus taeda): isolation and characterization of cDNA clones. Physiol Plant 97:139-148

Cheung F, Town CD (2007) A BAC end view of the Musa acuminata genome. BMC Plant Biol 7:29

De Langhe E, de Maret P (1999) Tracking the banana: its significance in early agriculture. In: Gosden C, Hather J (eds) The prehistory of food. Appetites for change. Routledge, London and New York, pp 377-396

De Langhe E, Hřibová E, Carpentier S, Doležel J, Swennen R (2010) Did backcrossing contribute to the origin of hybrid edible bananas? Ann Bot 106:849-857

Frankel N, Hasson E, Iusem ND, Rossi MS (2003) Adaptive evolution of the water stress-induced gene Asr2 in Lycopersicon species dwelling in arid habitats. Mol Biol Evol 20:1955-1962

Frankel N, Carrari F, Hasson E, Iusem ND (2006) Evolutionary history of the Asr gene family. Gene 378:74-83

Frankel N, Nunes-Nesi A, Balbo I, Mazuch J, Centeno D, Iusem ND, Fernie AR, Carrari F (2007) ci21A/Asr1 expression influences glucose accumulation in potato tubers. Plant Mol Biol 63:719730

Gilad A, Amitai-Zeigerson H, Bar-Zvi D, Scolnik PA (1997) ASR1, a tomato water-stress regulated gene: genomic organization, developmental regulation and DNA-binding activity. Acta Hort 447:447-454

Giombini MI, Frankel N, Iusem ND, Hasson E (2009) Nucleotide polymorphism in the drought responsive gene Asr2 in wild populations of tomato. Genetica 136:13-25

Ingram J, Bartels D (1996) The molecular basis of dehydration tolerance in plants. Annu Rev Plant Physiol Plant Mol Biol 47:377-403

Iusem ND, Bartholomew DM, Hitz WD, Scolnik PA (1993) Tomato (Lycopersicon esculentum) transcript induced by water deficit and ripening. Plant Physiol 102:1353-1354

Kalifa Y, Gilad A, Konrad Z, Zaccai M, Scolnik PA, Bar-Zvi D (2004) The water- and salt-stress-regulated Asrl (abscisic acid stress ripening) gene encodes a zinc-dependent DNA-binding protein. Biochem J 381:373-378

Konrad Z, Bar-Zvi D (2008) Synergism between the chaperone-like activity of the stress regulated ASR1 protein and the osmolyte glycine-betaine. Planta 227:1213-1219

Larkin MA, Blackshields G, Brown NP, Chenna R, McGettigan PA, McWilliam H, Valentin F, Wallace IM, Wilm A, Lopez R, Thompson JD, Gibson TJ, Higgins DG (2007) Clustal W and Clustal X version 2.0. Bioinformatics 23:2947-2948

Lescot M, Piffanelli P, Ciampi A, Ruiz M, Blanc G, Leebens-Mack J, da Silva F, Santos C, D'Hont A, Garsmeur O, Vilarinhos A, Kanamori H, Matsumoto T, Ronning C, Cheung F, Haas B, Althoff R, Arbogast T, Hine E, Pappas G, Sasaki T, Souza M, Miller R, Glaszmann J-C, Town C (2008) Insights into the Musa genome: syntenic relationships to rice and between Musa species. BMC Genomics 9:58

Liu HY, Dai JR, Feng DR, Liu B, Wang HB, Wang JF (2010) Characterization of a novel plantain Asr gene, MpAsr, that is regulated in response to infection of Fusarium oxysporum f. sp. cubense and abiotic stresses. J Integr Plant Biol 52:315-323

Maskin L, Gudesblat GE, Moreno JE, Carrari FO, Frankel N, Sambade A, Rossi M, Iusem ND (2001) Differential expression of the members of the Asr gene family in tomato (Lycopersicon esculentum). Plant Sci 161:739-746

Maskin L, Frankel N, Gudesblat G, Demergasso MJ, Pietrasanta LI, Iusem ND (2007) Dimerization and DNA-binding of ASR1, a small hydrophilic protein abundant in plant tissues suffering from water loss. Biochem Biophys Res Commun 352:831-835

Maskin L, Maldonado S, Iusem ND (2008) Tomato leaf spatial expression of stress-induced Asr genes. Mol Biol Rep 35:501505

Nelson A, Ploetz R, Kepler A (2006) Musa species (banana and plantain), ver. 2.2. In: Elevitch C (ed) Species profiles for Pacific Island agroforestry. Permanent agricultural resources (PAR). Holualoa, Hawai'i

Padmanabhan V, Dias DM, Newton RJ (1997) Expression analysis of a gene family in loblolly pine (Pinus taeda L.) induced by water deficit stress. Plant Mol Biol 35:801-807

Perrier X, Bakry F, Carreel F, Jenny C, Horry J, Lebot V, Hippolyte I (2009) Combining biological approaches to shed light on the evolution of edible bananas. Ethnobot Res \& Appl 7:199-216

Philippe R, Courtois B, McNally KL, Mournet P, El-Malki R, Le Paslier MC, Fabre D, Billot C, Brunel D, Glaszmann JC, This D (2010) Structure, allelic diversity and selection of Asr genes, candidate for drought tolerance, in Oryza sativa L. and wild relatives. Theor Appl Genet 121:769-787

Rajjou L, Belghazi M, Huguet R, Robin C, Moreau A, Job C, Job D (2006) Proteomic investigation of the effect of salicylic acid on Arabidopsis seed germination and establishment of early defense mechanisms. Plant Physiol 141:910-923

Riccardi F, Gazeau P, de Vienne D, Zivy M (1998) Protein changes in response to progressive water deficit in maize. Quantitative variation and polypeptide identification. Plant Physiol 117:12531263

Rossi M, Lijavetzky D, Bernacchi D, Hopp HE, Iusem N (1996) Asr genes belong to a gene family comprising at least three closely linked loci on chromosome 4 in tomato. Mol Gen Genet 252: 489-492

Rossi M, Carrari F, Cabrera-Ponce JL, Vazquez-Rovere C, HerreraEstrella L, Gudesblat G, Iusem ND (1998) Analysis of an abscisic acid (ABA)-responsive gene promoter belonging to the Asr gene family from tomato in homologous and heterologous systems. Mol Gen Genet 258:1-8

Roux N, Baurens FC, Dolezel J, Hribova E, Heslop-Harrison P, Town C, Sasaki T, Matsumoto T, Aert R, Remy S, Souza MT Jr, Lagoda P (2008) Genomics of banana and plantain (Musa spp.), major staple crops in the tropics. In: Moore PH, Ming R (eds) Genomics of tropical crop plants. Springer, New York, pp 83111

Santos CMR, Martins NF, Horberg HM, de Almeida ER, Coelho MC, Togawa RC, da Silva FR, Caetano AR, Miller RN, Souza MT Jr (2005) Analysis of expressed sequence tags from Musa acuminata ssp. burmannicoides, var. Calcutta 4 (AA) leaves submitted to temperature stresses. Theor Appl Genet 110:1517-1522

Santos CMR, Martins NF, Melo de Araújo M, da Silva FRM, Togawa R, Souza MT Jr (2009) Screening of the DATA Musa database for orthologs of genes known to be related to biotic and abiotic stresses to identify and characterise candidate genes. Acta Hort 828:341-352 
Seki M, Narusaka M, Abe H, Kasuga M, Yamaguchi-Shinozaki K, Carninci P, Hayashizaki Y, Shinozaki K (2001) Monitoring the expression pattern of 1,300 Arabidopsis genes under drought and cold stresses by using a full-length cDNA microarray. Plant Cell 13:61-72

Shinozaki K, Yamaguchi-Shinozaki K (2007) Gene networks involved in drought stress response and tolerance. J Exp Bot $58: 221-227$

Strosse H, Schoofs H, Panis B, Andre E, Reyniers K, Swennnen R (2006) Development of embryonic cells suspensions from shoot meristematic tissue in bananas and plantains (Musa spp.). Plant Sci 170:104-112

Thomas JE, Turner D, Eamus D (1998) Independent effects of the environment on the leaf gas exchange of three banana (Musa spp.) cultivars of different genomic constitution. Scient Hort 75:41-57

Vaidyanathan CP, Kuruvilla S, Thomas DS (1999) Characterization and expression pattern of an abscisic acid and osmotic stress responsive gene from rice. Plant Sci 140:21-30
Vandesompele J, De Preter K, Pattyn F, Poppe B, Van Roy N, De Paepe A, Speleman F (2002) Accurate normalization of real-time quantitative RT-PCR data by geometric averaging of multiple internal control genes. Genome Biol 3:RESEARCH0034

Wang H-J, Hsu C-M, Jauh GY, Wang CS (2005) A lily pollen ASR protein localizes to both cytoplasm and nuclei requiring a nuclear localization signal. Physiol Plant 123:314-320

Xiong L, Zhu JK (2002) Molecular and genetic aspects of plant responses to osmotic stress. Plant Cell Environ 25:131-139

Yang CY, Chen YC, Jauh GY, Wang CS (2005) A lily ASR protein involves abscisic acid signaling and confers drought and salt resistance in Arabidopsis. Plant Physiol 139:836-846

Yazaki J, Shimatani Z, Hashimoto A, Nagata Y, Fujii F, Kojima K, Suzuki K, Taya T, Tonouchi M, Nelson C, Nakagawa A, Otomo Y, Murakami K, Matsubara K, Kawai J, Carninci P, Hayashizaki Y, Kikuchi S (2004) Transcriptional profiling of genes responsive to abscisic acid and gibberellin in rice: phenotyping and comparative analysis between rice and Arabidopsis. Physiol Genomics 17:87-100 